\title{
Medial Prefrontal Cortex Neural Plasticity, Orexin Receptor 1 Signaling, and Connectivity with the Lateral Hypothalamus Are Necessary in Cue-Potentiated Feeding
}

\author{
Sindy Cole ${ }^{\star}$ Sara E. Keefer, ${ }^{\star}$ Lauren C. Anderson, and Gorica D. Petrovich \\ Department of Psychology, Boston College, Chestnut Hill, Massachusetts, 02467-3007
}

Cognitive processes contribute to the control of feeding behavior and help organism's survival when they support physiological needs. They can become maladaptive, such as when learned food cues drive feeding in the absence of hunger. Associative learning is the basis for cue-driven food seeking and consumption, and behavioral paradigms with Pavlovian cue-food conditioning are well established. Yet, the neural mechanisms underlying circuit plasticity across cue-food learning, cue memory recall, and subsequent food motivation are unknown. Here, we demonstrated the medial prefrontal cortex (mPFC) is a site of learning-induced plasticity and signaling of the neuropeptide orexin within the mPFC mediates cue potentiated feeding (CPF). First, using a marker of neuronal activation, $c$-fos, we confirmed that the mPFC is activated during CPF. Next, to assess whether the same mPFC neuronal ensemble is activated during cue-food learning and later CPF, we used the Daun02 chemogenetic inactivation method in $c$-fos-lac $Z$ transgenic male and female rats. Selective inactivation of the mPFC neurons that were active during the last cue-food training session abolished CPF during test, demonstrating that the MPFC is a site of plasticity. We postulated that integration of food cue memory and feeding motivation requires MPFC communications with lateral hypothalamus and showed that disconnection of that system abolished CPF. Then we showed that lateral hypothalamus orexin-producing neurons project to the mPFC. Finally, we blocked orexin receptor 1 signaling in the mPFC and showed that it is a neuromodulator necessary for the cue-driven consumption. Together, our findings identify a causal function for the MPFC in the cognitive motivation to eat.

Key words: appetite; learning; obesity; orexin; plasticity; prefrontal cortex

Significance Statement

Obesity has reached epidemic proportions, and the associated health consequences are serious and costly. The causes of obesity are complex because, in addition to physiological energy and nutrient needs, environmental cues can drive feeding through hedonic and cognitive processes. Learned food cues from the environment can powerfully stimulate appetite and food consumption in the absence of hunger. Using an animal model for cue-potentiated feeding, the current study determined the mPFC neuronal plasticity and neuropeptide orexin signaling are critical circuit and neurotransmitter mechanisms involved in this form of cognitive motivation to eat. These findings identify key targets for potential treatment of excessive appetite and overeating.

\section{Introduction}

The causes of obesity are complex because eating and body weight are not only controlled by internal, physiologic signals to

Received July 23, 2019; revised Jan. 6, 2020; accepted Jan. 9, 2020

Author contributions: S.C. and G.D.P. designed research; S.C., S.E.K., and L.C.A. performed research; S.C. and S.E.K. analyzed data; S.C. and G.D.P. wrote the first draft of the paper; S.C., S.E.K., L.C.A., and G.D.P. edited the paper.

This work was supported by National Alliance for Research on Schizophrenia and Depression Young Investigator Grant, Brain \& Behavior Research Foundation 22705 to S.C. and National Institute of Diabetes and Digestive and Kidney Diseases R01DK085721 to G.D.P. We thank Heather Mayer for technical support; Bret Judson and the Boston College Imaging Core for infrastructure and support; and Dr. Bruce Hope and Dr. Francois Vautier for advice and protocols regarding breeding c-fos-lacZ transgenic rats.

The authors declare no competing financial interests.

*S.C. and S.E.K. contributed equally to this work.

Correspondence should be addressed to Gorica D. Petrovich at gorica.petrovich@bc.edu. eat, but also by external, environmental, and social factors. Foodassociated cues are one important component of these external factors, and such cues have been shown to induce eating in sated children and adults (Birch et al., 1989; Cornell et al., 1989). We and others have modeled this behavior in rats using a cuepotentiated feeding (CPF) paradigm, originally developed by Weingarten (1983), to investigate the underlying neural circuits. Through Pavlovian conditioning, rats first learn to associate a neutral cue (e.g., a tone) with the delivery of a palatable food,

S. E. Keefer's present address: Department of Anatomy and Neurobiology, University of Maryland, School of Medicine, Baltimore, MD.

L. C. Anderson's present address: Preclinical Safety, Novartis Institutes for BioMedical Research, Cambridge, MA. https://doi.org/10.1523/JNEUROSCI.1803-19.2020

Copyright $\odot 2020$ the authors 
such that the cue becomes a signal for the food. Then, the ability of the cue to induce overeating is assessed in sated rats by presenting the cue, to which rats consume greater amounts of food compared with control cues, despite satiation (for review, see Petrovich, 2013). Prior work has identified the basolateral amygdala (BLA), lateral hypothalamus (LHA), and the mPFC as core components of the CPF neural network (see Discussion). It remains unknown whether these regions are similarly involved during the initial learning when cue-food associations are formed and later CPF tests when cue memory recall and induction of food motivation occurs.

The mPFC is necessary in flexible, goal-directed control of behavior (O'Doherty, 2011) and is important during both the initial cue-food learning, as well as the subsequent CPF. Using the neuronal activity marker, Fos, we found evidence of learninginduced plasticity across training in the MPFC with robust cuefood related activation evident in well-trained rats (Cole et al., 2015b). Neurotoxic lesions of the mPFC before training abolished contextual stimulus-driven consumption at test (Petrovich et al., 2007), whereas presentation of the learned food cue activated mPFC neurons (Petrovich et al., 2005). Therefore, the first two experiments tested whether the learning-induced plasticity within the mPFC is necessary in CPF. First, we confirmed that $\mathrm{CPF}$ tests increase the number of Fos-expressing neurons within the mPFC. Then, we used the Daun02 inactivation technique in c-fos-lac Z transgenic rats (Cruz et al., 2013; Koya et al., 2016) to determine whether the MPFC neuronal ensemble that was activated during the initial cue-food learning is necessary for $\mathrm{CPF}$.

The MPFC is anatomically well positioned to facilitate the integration of cue-food memory and feeding motivation (Balleine and O'Doherty, 2010; Moorman et al., 2015; Reppucci and Petrovich, 2016; Petrovich, 2018; Smith and Laiks, 2018). Stimulation of $\mu$-opioid or dopamine receptors within $\mathrm{mPFC}$ was found to induce feeding (Mena et al., 2011; Land et al., 2014), and $\mu$-opioid-induced consumption involved the LHA orexin (ORX) neurons (Mena et al., 2013). The PFC has extensive, bidirectional connections with the LHA, including inputs to the ORX neurons (for review, see Reppucci and Petrovich, 2016). ORX neurons are important during both cue-food learning and cue motivated actions, such as consumption during CPF (Petrovich et al., 2012; Cole et al., 2015b). Systemic blockade of ORX receptor 1 (ORXR1) prevented CPF and increased Fos induction in the mPFC (Cole et al., 2015a).

Based on this background and previously conceptualized ORX function in translating motivation into action (Mahler et al., 2014; Sakurai, 2014), we postulated that ORX signaling within mPFC may be critical in translating cue memory into feeding motivation. Therefore, we tested whether mPFC communication with the LHA is necessary for CPF and whether the ORX-R1 signaling within the MPFC is a key mediator. We used a contralateral disconnection technique with unilateral neurotoxic lesions to determine whether the LHA-mPFC system is necessary for $\mathrm{CPF}$. Then, we used retrograde tracing in combination with immunohistochemistry to confirm that ORX neurons contribute to an ascending $\mathrm{mPFC}$ projection, since that pathway has not been described in the literature. Finally, we determined a causal role for mPFC ORX signaling by antagonizing ORX-R1 within the mPFC during CPF tests.

\section{Materials and Methods}

Subjects

Experiments 1 and 2 used transgenic rats bred on a Sprague Dawley background in the animal facility at Boston College. Experiment 1 used
WT male and female Sprague Dawley rats, and Experiment 2 used $c$-foslacZ transgenic (RRID:RGD_9588581) male and female rats. The transgenic rats used to initiate the breeding program were a generous gift from Dr. Bruce Hope. For Experiments 3-5 male, Long-Evans rats were obtained from Charles River Laboratories (Experiments 3 and 5) and Taconic Biosciences (Experiment 4) and weighed 275-300 g at arrival. Rats were housed individually with ad libitum access to food and water, except when otherwise noted. The colony room was maintained at $21^{\circ} \mathrm{C}$ on a $12 \mathrm{~h}$ light/dark cycle (lights on at 06:00), and all behavioral testing was conducted during the light phase of the cycle. The housing and testing procedures were in accordance with the National Institute of Health Guidelines for the care and use of laboratory animals and approved by the Boston College Institutional Animal Care and Use Committee.

\section{Apparatus}

Training and testing were conducted in a set of behavioral chambers (Coulbourn Instruments) located in a room different from the colony room. Each chamber was located in a sound- and light-attenuating cubicle that was equipped with a ventilation fan $(55 \mathrm{~dB})$ and a video camera attached to a recording system in an adjacent room to record each training session (Coulbourn Instruments). The two stimuli used as cues were a $150 \mathrm{~s}$ tone $(75 \mathrm{~dB}, 2 \mathrm{kHz})$ and a $150 \mathrm{~s}$ presentation of the house light. The food stimulus consisted of 50 food pellets (5TUL, $45 \mathrm{mg}$; Test Diets; $3.4 \mathrm{kcal} / \mathrm{g} ; 20 \%$ protein, $13 \%$ fat, $67 \%$ carbohydrate) delivered to the food-cup of each chamber unless otherwise noted. The stimuli were controlled by the GraphicState 3.0 software system (Coulbourn Instruments).

\section{Surgery}

All surgeries were performed in a stereotaxic frame (Kopf Instruments) under anesthesia, using a mixture ( $1 \mathrm{ml} / \mathrm{kg}$ body weight) of ketamine (50 $\mathrm{mg} / \mathrm{ml})$ and xylazine $(10 \mathrm{mg} / \mathrm{ml})$. All incisions were closed with wound clips, and a triple antibiotic cream was applied. Following surgery, rats were given the analgesic rimadyl ( $2 \mathrm{mg} / 100 \mathrm{~g}$ body weight $)$ and allowed at least $5 \mathrm{~d}$ to recover before behavioral manipulations during which time they were monitored and weighed daily.

Cannula implantations (Experiments 2 and 5). Guide cannulae (23 gauge; Plastics One) were bilaterally implanted above the mPFC. The flat-skull coordinates used from bregma were as follows: AP, $3.00 \mathrm{~mm}$; $\mathrm{ML}, \pm 1.5 \mathrm{~mm}$; DV,$-4.30 \mathrm{~mm}$ with an angle of $10^{\circ}$. The cannulae were anchored to the skull with jeweler's screws and dental cement. Obturators were inserted into the guide cannulae and removed regularly to ensure patency of the cannulae.

Excitotoxic lesions (Experiment 3). Craniotomies were performed above each injection site with a drill, and a $1 \mu \mathrm{l} 32$-gauge Hamilton 'Neuros' syringe driven by a Quintessential Stereotaxic Injector (Stoelting) was used to make each injection. Rats received one $0.3 \mu$ injection into the $\mathrm{mPFC}$ and one $0.35 \mu$ injection into the LHA delivered at a rate of $0.1 \mu \mathrm{l} / \mathrm{min}$. Injections consisted of either $0.15 \mathrm{M}$ NMDA (Sigma Millipore) in PBS to produce lesions, or PBS alone. The flat-skull coordinates of the injections from bregma were as follows: for $\mathrm{mPFC}, \mathrm{AP}, 3.0$ $\mathrm{mm}$; ML, $0.7 \mathrm{~mm}$; DV, $-4.5 \mathrm{~mm}$; and for LHA, AP, $-2.7 \mathrm{~mm}$; ML, 1.3 $\mathrm{mm} ; \mathrm{DV},-8.9 \mathrm{~mm}$. Following each injection, the needle was left in place for $4 \mathrm{~min}$ to allow for diffusion of the injectate. Any drill holes in the skull were filled with bone wax before the incision was closed.

Fluoro-Gold (FG) deposits (Experiment 4). The bone above the mPFC was removed with a drill, and a unilateral injection of $40 \mathrm{nl}$ of $3 \%$ FG (Fluorochrome) (AP, $2.0 \mathrm{~mm}$; ML, $0.8 \mathrm{~mm}$; DV, $-4.7 \mathrm{~mm}$ ) was delivered using the syringe and injector described above. The FG was injected over $5 \mathrm{~min}$, with the needle left in place for an additional $2 \mathrm{~min}$. The drill hole was filled with bone wax before closure of the incision.

\section{Intracranial injections}

Before testing, rats were gently wrapped in a towel and had their obturators removed to familiarize them with the infusion procedure. For intracranial infusions, the obturators were removed, and injectors connected via polyethylene tubing to a $10 \mu$ l glass Hamilton syringe mounted on an infusion pump were inserted into the guide cannulae. The injectors projected an additional $1 \mathrm{~mm}$ ventral to the tip of the guide cannulae. Fol- 
lowing microinfusion, the obturators were reinserted, and the rats were returned to their home cage.

Drugs. For Experiment 2, Daun02 (Sequoia Research Products) was dissolved in 5\% DMSO (Sigma Millipore), 6\% Tween 80, and 89\% PBS, and administered at a dose of $2 \mu \mathrm{g} / 0.5 \mu \mathrm{l} /$ side. The solution for vehicle infusions included the same components but excluded the Daun02. For Experiment 5, the ORX-R1 antagonist SB-334867 (SB; Tocris Bioscience) was dissolved in DMSO and administered at a dose of $6.26 \mathrm{~mm} \mathrm{(2}$ $\mu \mathrm{g} / \mu \mathrm{l})$ and volume of $0.5 \mu \mathrm{l}$ into each hemisphere, based on prior work (Borgland et al., 2006; James et al., 2011). Vehicle infusions consisted of DMSO. All solutions were infused over $1 \mathrm{~min}$, with the injectors left in place for an additional $1 \mathrm{~min}$.

\section{Immunohistochemistry and analysis}

At the completion of each experiment, rats were anesthetized with tribromoethanol (312.5 mg/kg; $1.25 \mathrm{ml} / 100 \mathrm{~g}$ body weight, i.p.). In Experiments $1-4$, they were then transcardially perfused with $0.9 \%$ saline followed by ice-cold $4 \%$ PFA in $0.1 \mathrm{~m}$ borate buffer, $\mathrm{pH}$ 9.4. The brains were stored for $20-24 \mathrm{~h}$ at $4^{\circ} \mathrm{C}$ in the fixative with $12 \%$ sucrose and then rapidly frozen in hexanes cooled with dry ice and stored at $-80^{\circ} \mathrm{C}$. Frozen brains were cut into $40 \mu \mathrm{m}$ coronal sections using a sliding microtome (Leica Microsystems) and collected into serially adjacent series. One series of sections from each experiment were mounted onto chrome alum/gelatin-coated slides and stained with thionine for identification of cytoarchitectonic borders.

Single-labeled Fos neurons (Experiments 1 and 2). Immediately following slicing of the $\mathrm{MPFC}$, brain sections from one series were rinsed in potassium PBS (KPBS) and then incubated with anti-c-fos antibody raised in rabbit (1:30,000, Ab-5, PC38; Calbiochem) in a blocking solution of KPBS containing 2\% normal goat serum (S-1000; Vector Laboratories; RRID:AB_2336615) and 0.3\% Triton X-100 (Sigma Millipore) for $72 \mathrm{~h}$ at $4^{\circ} \mathrm{C}$ with gentle agitation. Sections were then brought to room temperature, rinsed with KPBS, incubated with biotinylated secondary antibody against rabbit (1:200, BA-1000; Vector Laboratories; RRID: AB_2313606) in the blocking solution for $90 \mathrm{~min}$, rinsed in KPBS, incubated in avidin biotin complex (PK-6100; Vector Laboratories; RRID: AB_2336819) for $90 \mathrm{~min}$, and again rinsed in KPBS. Neurons immunoreactive for Fos were visualized as brown after a 1 min incubation in DAB (SK-4100; Vector Laboratories; RRID:AB_2336382). Sections were then rinsed, mounted on SuperFrost slides (Thermo Fisher Scientific), dried at $40^{\circ} \mathrm{C}$, dehydrated through graded alcohols, cleared in xylenes, and coverslipped with DPX Mountant (Electron Microscopy Services).

Images of the Fos-stained and adjacent thionine-stained sections were acquired (10X magnification) with an Olympus BX51 light microscope attached to an Olympus DP72 camera using DP2-BSW software (Olympus America). Using ImageJ software (National Institutes of Health), the images were stacked and transformed to 8-bit grayscale, and automated counting was performed within the ROI consistently across sections and brains using the same criteria. For both Experiments 1 and 2, a rectangular template was used. The template used in Experiment 2 was smaller and centered below the ventral point of the injector tip to avoid any counting of extraneous Fos resulting from the cannula. The threshold for counting Fos-positive cells was determined from an area on each section with no specific labeling (background). Images were acquired bilaterally from mid rostrocaudal level (Atlas Level 8, $3.2 \mathrm{~mm}$ from bregma) (Swanson, 2004) for all analyses, and cell counts in the left and right hemispheres were summed for each rat, then averaged for each group, resulting in a mean total of Fos-labeled neurons.

Double-labeled Fos and $\beta$-gal neurons (Experiment 2). Free-floating sections of the mPFC were incubated with anti-c-fos antibody raised in rabbit (1:1000, 9F6, \#2250; Cell Signaling Technology; RRID: AB_2247211) and anti- $\beta$-gal antibody raised in mouse (1:2000, BG-02, ab1047, Abcam; RRID:AB_297212) in a blocking solution of KPBS containing $2 \%$ normal donkey serum (Jackson ImmunoResearch Laboratories) and $0.3 \%$ Triton X-100 (Sigma Millipore) for $72 \mathrm{~h}$ at $4^{\circ} \mathrm{C}$ with gentle agitation. Sections were then rinsed in KPBS and then incubated with secondary fluorescent antibodies: Alexa-488 anti-rabbit raised in donkey (1:200; A21206; Invitrogen; RRID:AB_141708) and Alexa-594 antimouse raised in donkey (1:200; A21203; Invitrogen; RRID:AB_141633) in the blocking solution for $1 \mathrm{~h}$ in the dark. Tissue was rinsed in KPBS and then immediately mounted in semidarkness onto SuperFrost slides, airdried, coverslipped with Vectashield HardSet Mounting Medium with DAPI (H-1500; Vector Laboratories; RRID:AB_2336788), and stored at $4^{\circ} \mathrm{C}$ until analysis.

Images were captured using the $20 \times$ objective on a Carl Zeiss AxioImager Z2 fluorescence microscope with an attached Hamamatsu ORCA R2 camera controlled through Zen software (version 2.3). Images were acquired bilaterally in the mPFC (Level 8, 3.20 from bregma) and pseudo-colored with green for Fos, red for $\beta$-Gal, and blue for DAPI (nuclear counterstain). Counts were performed manually from the triple-merged images, and bilateral counts were summed for each rat. The percentage of $\beta$-gal neurons that were labeled with Fos (doublelabeled) was calculated.

Lesion verification using $\mathrm{NeuN}$ (Experiment 3). Immediately following slicing of the forebrain, including the $\mathrm{MPFC}$ and LHA regions, sections from one series were incubated for $1 \mathrm{~h}$ in a blocking solution (KPBS containing normal horse serum, Triton X-100, and milk), and then incubated with mouse antiserum against NeuN (1:1000, MAB377; Millipore; RRID:AB_2298772) in the blocking solution for $72 \mathrm{~h}$ at $4^{\circ} \mathrm{C}$. Sections were subsequently rinsed with KPBS, normal horse serum, and milk, incubated with biotinylated secondary antibody against mouse (1: 500, BA-2001; Vector Laboratories; RRID:AB_2336180) in the blocking solution for $45 \mathrm{~min}$, rinsed in KPBS, incubated in avidin biotin complex (PK-6100; Vector Laboratories; RRID:AB_2336819) for $45 \mathrm{~min}$, and rinsed again in KPBS. Nuclei labeled for NeuN were visualized as gray with nickel-intensified DAB (SK-4100; Vector Laboratories; RRID: AB_2336382). Sections were rinsed, mounted on SuperFrost slides, dried at $40^{\circ} \mathrm{C}$, dehydrated through graded alcohols, cleared in xylenes, and coverslipped with DPX Mountant. A second series of sections were mounted from KPBS onto gelatin-coated slides and stained with thionine for secondary verification of lesion extent.

NeuN-stained sections were examined under a light microscope (Olympus, BX51), and the extent of each lesion was mapped using the rat brain atlas of Swanson (2004). Any subject with insufficient damage to either the mPFC or LHA $(<50 \%)$ was excluded from further analyses ( $n=20$ exclusions).

FG deposit confirmation (Experiment 4). Immediately following slicing of the MPFC, free-floating sections were immunohistochemically processed similar to the NeuN (above), except that the primary and secondary antibodies used were anti-FG raised in rabbit (1:5000, AB153, Millipore; RRID:AB_90738) and biotinylated secondary against rabbit (1:500, BA-1000, Vector Laboratories; RRID:AB_2313606), respectively. Additionally, FG was visualized as brown with DAB (SK-4100; Vector Laboratories; RRID:AB_2336382).

FG injection sites were examined using the $10 \times$ objective on an Olympus BX51 light microscope attached to an Olympus DP72 camera using DP2-BSW software (Olympus America). Location and extent of injections were determined based on adjacent thionine-stained tissue and were transposed onto rat brain atlas templates (Swanson, 2004). Injections predominantly confined to the MPFC were included $(n=5)$.

Double-labeled FG and ORX neurons (Experiment 4). Free-floating sections of the LHA were incubated with anti-FG antibody raised in rabbit (1:5000, AB153; Millipore; RRID:AB_90738) and anti-ORX antibody raised in goat (1:2000, sc-8070; Santa Cruz Biotechnology; RRID; AB_653610) in a blocking solution of KPBS containing 2\% normal donkey serum (Jackson ImmunoResearch Laboratories) and 0.3\% Triton X-100 (Sigma Millipore) for $72 \mathrm{~h}$ at $4^{\circ} \mathrm{C}$ with gentle agitation. Sections were then rinsed in KPBS and then incubated with secondary fluorescent antibodies: Alexa-488 anti-rabbit raised in donkey (1:200; A21206; Invitrogen; RRID:AB_141708) and Alexa-546 anti-goat raised in donkey (1: 200; A11056; Invitrogen; RRID:AB_142628) in the blocking solution for $1 \mathrm{~h}$ in the dark. Tissue was rinsed in KPBS and then immediately mounted in semidarkness onto SuperFrost slides, air-dried, coverslipped with Vectashield HardSet Mounting Medium, and stored at $4^{\circ} \mathrm{C}$ until analysis.

Analysis was conducted from images acquired on the ipsilateral side of the FG deposit throughout the ORX field in the LHA (Atlas Levels 26-32, -1.78 through -3.90 from bregma). Images were pseudo-colored with 
green for FG, red for ORX, and blue for DAPI (nuclear counterstain). Counts were performed manually from the triple-merged images, with single images consulted as needed. For each image, the number of FGpositive cells, ORX-positive neurons, and FG + ORX-positive cells (visualized as yellow) were counted. For each brain, these counts were then summed, and the percentage of FG-labeled neurons that were ORXpositive (double-labeled) and the percentage of ORX-positive neurons that were FG-labeled were calculated.

\section{Cannula placement verification (Experiments 2 and 5)}

For Experiment 2, the thionine-stained tissue was examined under light microscopy, and cannula placements were mapped using the rat brain atlas of Swanson (2004). For Experiment 5, at the completion of the experiment, brains were removed, and the mPFC was blocked and immersed in $4 \%$ PFA in $0.1 \mathrm{~m}$ borate buffer, $\mathrm{pH} 9.4$, for 1 week at $4^{\circ} \mathrm{C}$. The tissue was then stored for $20-24 \mathrm{~h}$ at $4^{\circ} \mathrm{C}$ in the fixative with $12 \%$ sucrose, and then cut into $40 \mu \mathrm{m}$ coronal sections using a sliding microtome and collected into KPBS and stored at $4^{\circ} \mathrm{C}$. The sections were mounted onto chrome alum/gelatin-coated slides, dried at $40^{\circ} \mathrm{C}$, dehydrated through graded alcohols, stained with thionine, cleared in xylenes, and coverslipped with DPX Mountant. Cannula placements were verified as in Experiment 2.

\section{Behavioral procedures}

For all experiments, on the day before the start of training, chow was removed from the home cages, and all rats received $1 \mathrm{~g}$ of the training food pellets in their home cage to familiarize them with the pellets. Rats remained food-restricted throughout training, with access to chow for $1 \mathrm{~h}$ each day following the final training session.

Training. Training took place across 8 consecutive days, and each day consisted of a CS ${ }^{+}$(Food) session, a CS ${ }^{-}$(No Food) session, or both $\mathrm{CS}^{+}$and $\mathrm{CS}^{-}$sessions with an intersession interval ranging from 2 to $3.5 \mathrm{~h}$, which varied across days (see description of specific experiments below). Each session was $30 \mathrm{~min}$ in duration. During a Food session, rats received two presentations of one cue (e.g., tone), which overlapped and coterminated with delivery of the US (food). During a No Food session, rats received two presentations of the other cue (e.g., light), which overlapped with the sound of the food pump, but no food was delivered. When training consisted of both types of sessions, the order of Food and No Food sessions was counterbalanced across days. At the completion of the final training session, all rats were returned to ad libitum chow access for $3 \mathrm{~d}$ before testing began.

Testing. Testing consisted of a baseline consumption test followed by a cue test. For the baseline test, rats were placed into the behavioral chamber for two consecutive $10 \mathrm{~min}$ periods, each with access to $7 \mathrm{~g}$ of food pellets within the food-cup. After each test period, rats were briefly removed from the chamber, and any remaining pellets were collected to be weighed later. Following completion of the baseline test, rats were placed back into the behavioral chamber for a cue test session ( $\sim 10 \mathrm{~min})$, during which they received three presentations of the CS, each coterminated with delivery of 50 food pellets. All rats were then returned to the colony room, and any remaining food pellets were counted.

\section{Experimental design and statistical analysis}

Experiment 1: Fos expression in mPFC during cue-potentiated feeding. The design of Experiment 1 is displayed in Figure 1A. During training, rats in the paired group $(n=8)$ received $\mathrm{CS}^{+}$training with the tone (tone-food pairings), whereas rats in the unpaired group $(n=8)$ received $\mathrm{CS}^{-}$ training (tone alone) followed by later food pellet delivery in the home cage. After training, rats were returned to ad libitum feeding for $3 \mathrm{~d}$, following which they all received a single test session. To align the timing of peak Fos expression with consumption during the cue test, rather than consumption during the baseline period, all rats were returned to the colony room for $30 \mathrm{~min}$ in between the baseline and cue tests. Ninety minutes from the beginning of the cue test, rats were perfused, and the brains removed for later immunohistochemistry staining for Fos detection.

Experiment 2: Daun02 inactivation of $m P F C$ neurons during cuepotentiated feeding. In $c$-fos-lac $Z$ transgenic rats, the protein $\beta$-galactosidase $(\beta$-gal) is expressed under the control of the Fos promoter; therefore, $\beta$-gal is selectively expressed in recently activated neurons.
When the prodrug Daun02 is injected into discrete brain regions, the $\beta$-gal present in local Fos-expressing neurons converts Daun02 into the cytotoxin daunorubicin, resulting in neuronal inactivation through disruption of voltage-dependent calcium channels and apoptotic mechanisms (Cruz et al., 2013; Koya et al., 2016). The design of this experiment is displayed in Figure 2A. c-fos-lac $Z$ transgenic rats underwent surgery to implant cannulae bilaterally above the mPFC. Following recovery, food restriction began, and training commenced. All rats received $7 \mathrm{~d}$ of $\mathrm{CS}^{+}$training with the tone. On day 8 ("induction day"), $90 \mathrm{~min}$ from the beginning of the training session, rats were separated into two groups and received intracranial infusions into the mPFC of either Daun02 $(n=4)$ or vehicle $(n=5)$. Rats remained in their home cages for $3 \mathrm{~d}$ to allow Daun02 to take full effect, during which time they had ad libitum access to food. All rats then received a single test session consisting of a baseline test and a cue test separated by $30 \mathrm{~min}$. Ninety minutes from the beginning of the cue test, rats were perfused and the brains processed for single-labeled Fos as well as Fos $+\beta$-gal doublelabeling detection.

Experiment 3: effect of mPFC-LHA system disconnection on cuepotentiated feeding. The design of Experiment 3 is displayed in Figure $3 A$. Rats received unilateral neurotoxic lesions of the mPFC and LHA in opposite hemispheres (contralateral; $n=7$ ), the same hemisphere (ipsilateral; $n=7$ ), or contralateral vehicle infusions ( $\operatorname{sham;} n=6$ ). Following recovery, rats began food restriction and received training consisting of daily $\mathrm{CS}^{+}$and $\mathrm{CS}^{-}$sessions. Rats then returned to ad libitum feeding and were subsequently tested for consumption during presentations of the $\mathrm{CS}^{+}$and $\mathrm{CS}^{-}$cues separately, in a counterbalanced order, across two consecutive days. Each test consisted of a baseline test followed immediately by the cue test. Following the final test, rats were perfused and the brains processed for NeuN detection to confirm the location and extent of the excitotoxic lesions.

Experiment 4: mapping of the LHA orexinergic projection to mPFC. To confirm and characterize the topography of mPFC inputs from ORX neurons, the retrograde tracer FG was injected unilaterally into the mPFC. Two weeks following surgery, rats were perfused, and the brains were processed for detection of neurons with the FG deposit and doublelabeled FG + ORX neurons within the LHA. In rats with successful placements of FG, based on location and spread in $\operatorname{mPFC}(n=5)$, we mapped LHA neurons with the FG (neurons that give rise to LHA-mPFC pathways). Additionally, we mapped neurons with FG + ORX labeling to determine whether the LHA-mPFC pathway originated from ORX neurons.

Experiment 5: effect of blocking ORX-R1 signaling in $M P F C$ on cuepotentiated feeding. The design of Experiment 5 is displayed in Figure $5 \mathrm{~A}$. Rats underwent surgery to implant cannulae bilaterally in the mPFC. Following recovery, rats received training consisting of daily $\mathrm{CS}^{+}$and $\mathrm{CS}^{-}$sessions, under food restriction. Rats then returned to ad libitum feeding and were subsequently tested for consumption during presentations of the $\mathrm{CS}^{+}$or $\mathrm{CS}^{-}$, in a counterbalanced order, across $3 \mathrm{~d}$ (one test per day and a rest day in between). On each test day, rats first received the baseline test drug-free to ensure that all rats were well sated before the cue feeding test. Immediately following the baseline test, rats received an injection into the mPFC of either SB-334867 $(n=7)$ or vehicle $(n=7)$, and after 10-15 min were returned to the conditioning chambers where they received the cue test. Animals received identical infusions before each cue test (SB-SB or Veh-Veh).

\section{Statistical analysis}

For training, the dependent variable, the primary measure of learning, was the percentage of time rats expressed food-cup behavior during CS presentations. Food-cup behavior was defined as distinct nose pokes into the food-cup or rats standing in front of and directly facing the food-cup. Behavior was scored every $1.25 \mathrm{~s}$ during the CS, with the number of food-cup observations summed and converted to a percentage. For testing, the dependent variable was the amount of food pellets consumed during the baseline tests in grams and during the cue tests as number of pellets. All analyses were conducted using either mixed-model ANOVAs, or $t$ tests, where appropriate. Statistical analyses were performed using 
SPSS (version 22, IBM), and statistical significance was set at $p \leq 0.05$. Data are presented as mean \pm SEM, unless otherwise noted.

\section{Results}

Experiment 1: Fos expression in MPFC during cue-potentiated feeding

Here we examined Fos induction in the $\mathrm{mPFC}$ to determine whether cue-induced eating in sated rats is associated with neuronal activation of the prelimbic (PL) and infralimbic (ILA) cortical areas.

\section{Training}

Rats in the paired group increased foodcup responding during CS presentations across training (Fig. $1 B$ ). A group $\times$ training day repeated-measures ANOVA showed a main effect of day $\left(F_{(1,14)}=20.602, p=\right.$ $0.001)$, a main effect of group $\left(F_{(1,14)}=\right.$ 16.851, $p=0.001)$, and a significant group $\times$ day interaction $\left(F_{(1,14)}=7.453\right.$, $p=0.016)$. Follow-up tests confirmed that rats in the paired group showed significantly greater responding during day 8 compared with day $1\left(t_{(7)}=5.244, p=\right.$ $0.001)$, but unpaired rats did not $\left(t_{(7)}=\right.$ $1.255, p=0.25$ ). There were no differences in responding during pre-CS at either the beginning $\left(t_{(14)}=0.239, p=\right.$ $0.814)$ or end $\left(t_{(14)}=0.990, p=0.339\right)$ of training.

Test

There were no differences between groups in consumption during the baseline test $\left(t_{(14)}=0.341, p=0.738\right)$. During the cue test (Fig. $1 C$ ), rats in the paired group showed cue-potentiated feeding; they consumed significantly more pellets during CS presentations than rats in the unpaired group $\left(t_{(14)}=2.314, p=0.036\right)$.

\section{Fos induction}

We observed that cue-potentiated feeding was associated with increased Fos expression in the mPFC (Fig. 1D). Representative images of Fos-stained mPFC tissue are shown in Figure 1E. Rats in the paired group had significantly greater Fos expression than rats in the unpaired group in both the PL $\left(t_{(14)}=2.700, p=\right.$ $0.017)$, and the ILA $\left(t_{(14)}=2.906, p=0.011\right)$.

\section{Experiment 2: Daun02 inactivation of $\mathrm{mPFC}$ neurons during cue-potentiated feeding}

Experiment 1 demonstrated that the mPFC neurons are activated when overeating was driven by a learned food cue in sated rats. Experiment 2 used the Daun02 inactivation method to determine whether the same $\mathrm{mPFC}$ neuronal ensemble that was originally activated during cue-food learning needs to be activated during cue-potentiated feeding.

\section{Training and induction day}

All rats increased their food-cup responding during CS presentations across training (Fig. $2 B$ ). A group $\times$ training day repeatedmeasures ANOVA showed a main effect of day $\left(F_{(1,7)}=45.139\right.$, $p=0.0002)$, but no effect of group $\left(F_{(1,7)}=0.027, p=0.874\right)$, or
Behavioral Procedure

\begin{tabular}{|c|c|} 
Home Cage & Test \\
\hline $\begin{array}{c}\text { ad lib chs } \\
\text { Baseline and } \\
\text { Cue Consumption }\end{array}$
\end{tabular}

C

Training

Test

Cue Consumption

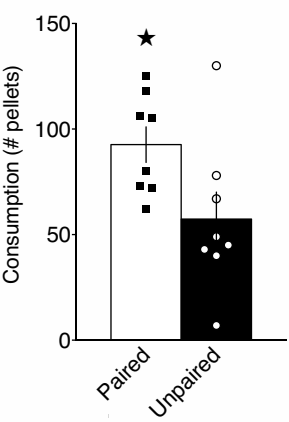

E

Test

Fos Induction

Fos labeling

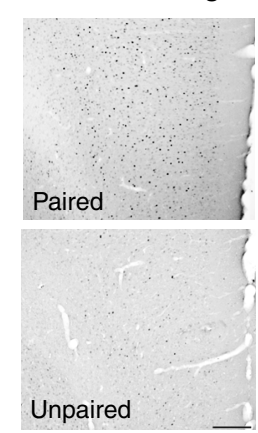

Figure 1. Fos expression in $\mathrm{mPFC}$ during cue-potentiated feeding. $\boldsymbol{A}$, Experimental design. $\boldsymbol{B}$, Conditioned responses during the first and last training sessions. $C$, Food consumption during cue tests. $\boldsymbol{D}$, Number of Fos-positive neurons in PL and ILA. $\boldsymbol{E}$, Representative images of Fos labeling in PL, scale bar $=200 \mu \mathrm{m}$. B - D, Data are mean \pm SEM. ${ }^{*} p<0.05$.

group $\times$ day interaction $\left(F_{(1,7)}=0.168, p=0.694\right)$. On induction day, there were no differences in responding between the two groups before receiving Daun02 or vehicle infusions following the session $\left(t_{(7)}=0.096, p=0.926\right)$. There were no differences in responding during pre-CS at either the beginning of training $\left(t_{(7)}=1.589, p=\right.$ $0.156)$ or on induction day $\left(t_{(7)}=1.610, p=0.152\right)$.

Test

Three days after induction, rats were tested in consumption tests during cue presentations following a baseline test. There was no difference in consumption between Daun02 and vehicle groups $\left(t_{(7)}=1.507, p=0.176\right)$ during the baseline test. Consumption during the cue test (tone presentations) is shown in Figure 2C. Rats that received Daun02 into the MPFC following the induction cue-food session showed impaired cue-potentiated feeding at test, as they consumed significantly fewer pellets than those that had received vehicle treatment $\left(t_{(7)}=3.522, p=0.010\right)$.

Fos and $\beta$-gal analysis

Location of the injector tips in MPFC are displayed in Figure 2D. Rats that received Daun02 injections following the induction cuefood session had a fewer number of Fos-positive neurons at test in the mPFC. Analyses showed a significant difference in Fos expression between the Daun02 and vehicle groups in the PL $\left(_{(7)}=2.513, p=\right.$ 
A

Behavioral Procedure

\begin{tabular}{|c|c|c|c|c|}
\hline Surgery & Training & Induction & Home Cage & Test \\
\hline & $\begin{array}{c}7 \text { sessions } \\
2 \mathrm{CS}+\end{array}$ & $\begin{array}{c}1 \text { session } \\
2 C S+\end{array}$ & $\begin{array}{c}3 \text { days } \\
\text { ad lib chow }\end{array}$ & $\begin{array}{c}1 \text { session } \\
\text { Baseline and } \\
\text { Cue Consumption }\end{array}$ \\
\hline
\end{tabular}

B

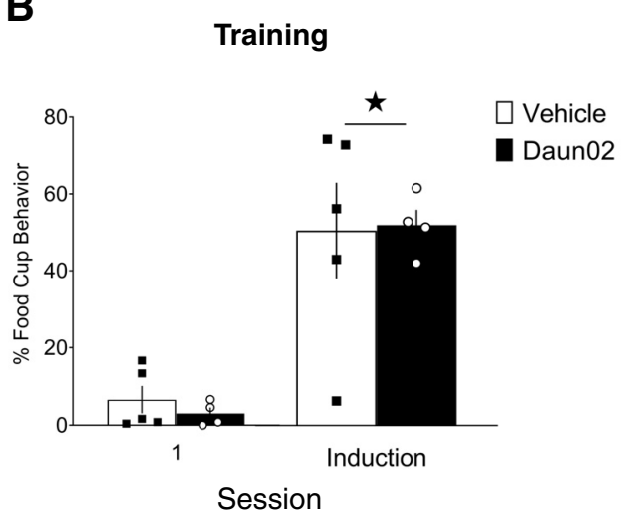

E

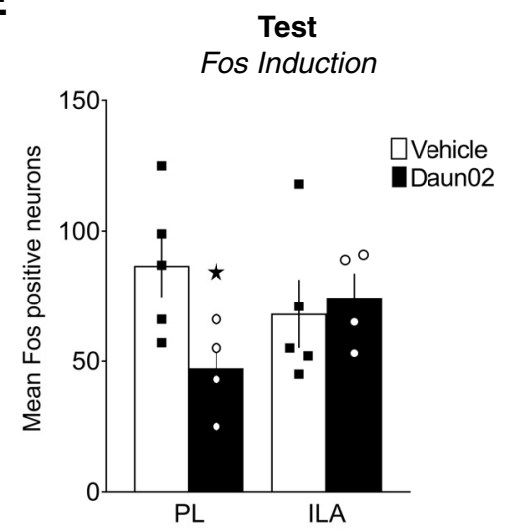

C

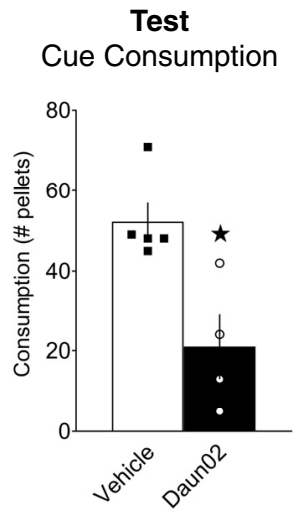

F

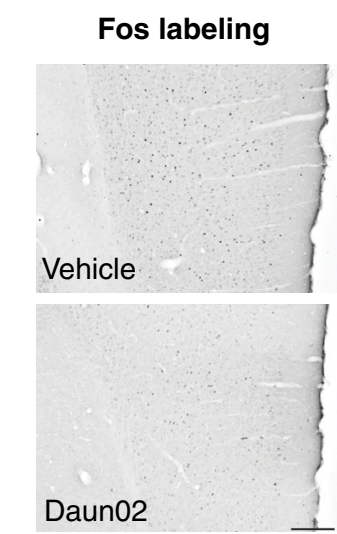

D

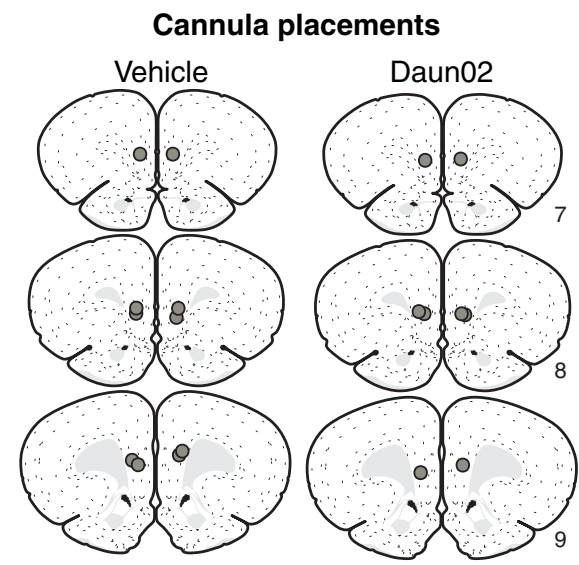

G

Fos and $\beta$-Gal double-labeling
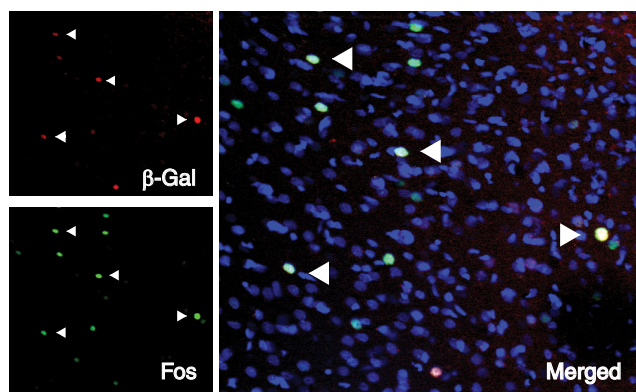

Figure 2. Daun02 inactivation of $\mathrm{mPFC}$ neurons during cue-potentiated feeding. $\boldsymbol{A}$, Experimental design. $\boldsymbol{B}$, Conditioned responses during the first and last (induction) training sessions. $C$, Food consumption during cue tests. $\boldsymbol{D}$, Cannula placements. $\boldsymbol{E}$, Number of Fos-positive neurons in PL and ILA. $\boldsymbol{F}$, Representative images of Fos labeling in PL, scale bar $=200 \mu \mathrm{m}$. $\mathbf{G}$, Representative images of Fos, $\beta$-gal, and Fos $+\beta$-gal (Merged) labeling. $B, C, E$, Data are mean \pm SEM. ${ }^{*} p<0.05$.

0.04 ), but not the ILA $\left(t_{(7)}=0.370, p=0.722\right)$ (Fig. $\left.2 E\right)$. Representative images of Fos-stained mPFC tissue are shown in Figure $2 F$.

Quantification of Fos $+\beta$-gal double-labeled neurons found that $97 \%$ of $\beta$-gal neurons were also Fos-positive. This percentage of coexpression is comparable with prior studies (Bossert et al., 2012; Fanous et al., 2012; Funk et al., 2016). Representative images showing Fos and $\beta$-gal staining as well as Fos $+\beta$-gal overlap are shown in Figure $2 G$.

\section{Experiment 3: effect of mPFC-LHA system disconnection on} cue-potentiated feeding

We used a contralateral lesion placement design that bilaterally disconnected the LHA communications with the MPFC to test whether the LHA-mPFC system is required for cue-induced eating. Representative images of NeuN-stained tissue from lesion and sham groups are shown in Figure $3 B$. The extent and location of each lesion are represented in Figure $3 C$.
Training

All groups acquired discrimination between cues across training, as indicated by greater food-cup behavior during $\mathrm{CS}^{+}$presentations compared with $\mathrm{CS}^{-}$presentations (Fig. 3D). Furthermore, all groups showed an equivalent increase in discriminative foodcup responding $\left(\left[\mathrm{CS}^{+}\right]\right.$minus $\left.\left[\mathrm{CS}^{-}\right]\right)$from day 1 to day 8 of training. A group $\times$ training day repeated-measures ANOVA showed a main effect of day $\left(F_{(1,17)}=98.412, p<0.001\right)$, but no effect of group $\left(F_{(2,17)}=0.117, p=0.890\right)$, or group $\times$ day interaction $\left(F_{(2,17)}=3.097, p=0.071\right)$.

\section{Test}

There were no differences between groups in baseline consumption that occurred immediately before each cue test. A group $X$ test session repeated-measures ANOVA showed no effect of session $\left(F_{(1,17)}=0.201, p=0.660\right)$, group $\left(F_{(2,17)}=0.407, p=\right.$ $0.672)$, or group $\times$ session interaction $\left(F_{(1,17)}=1.155, p=\right.$ 
A

Behavioral Procedure

\begin{tabular}{|c|c|c|c|}
\hline Surgery & Training & Home Cage & Test \\
\hline & $\begin{array}{c}8 \text { CS+ sessions } \\
\text { (2 cue-food pairings) } \\
8 \text { CS- sessions } \\
\text { (2 cue only presentations) }\end{array}$ & $\begin{array}{c}3 \text { days } \\
\text { ad lib chow }\end{array}$ & $\begin{array}{c}1 \mathrm{CS}+\text { session } \\
\text { (Baseline }+ \text { Cue consumption) } \\
1 \text { CS- session } \\
\text { (Baseline }+ \text { Cue consumption) }\end{array}$ \\
\hline
\end{tabular}

B

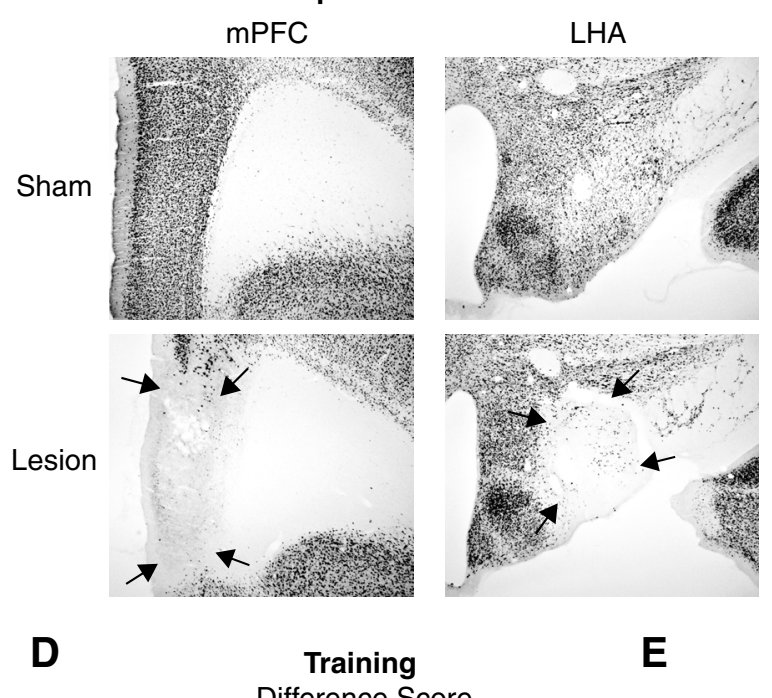

C

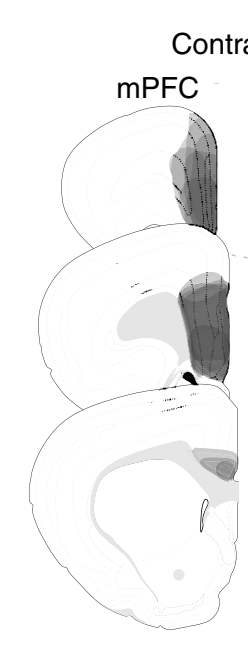

Test

Cue Consumption
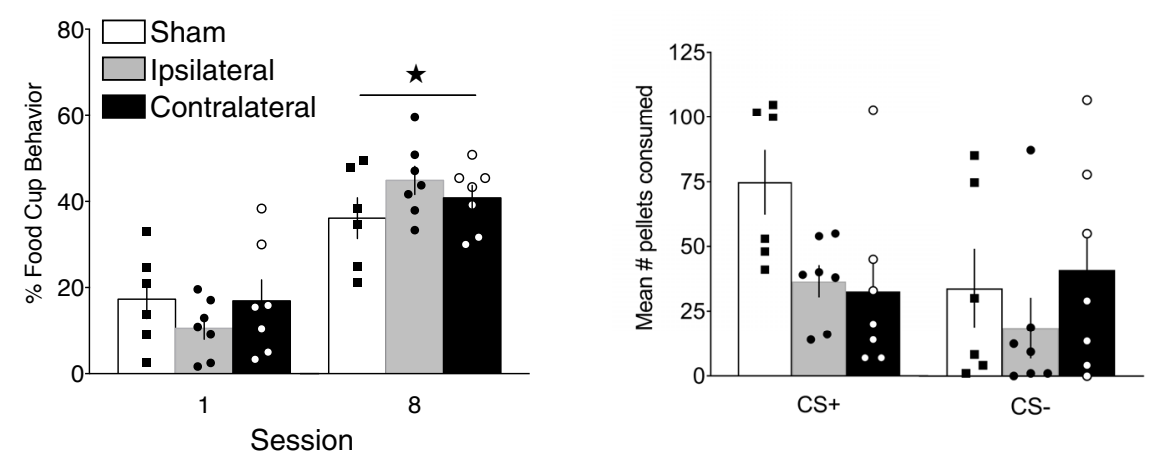

Extent of lesions

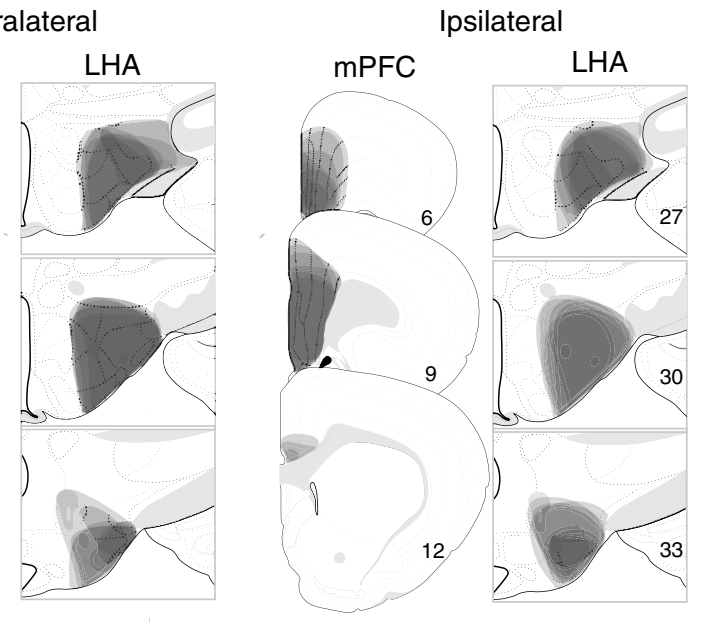

F

Test

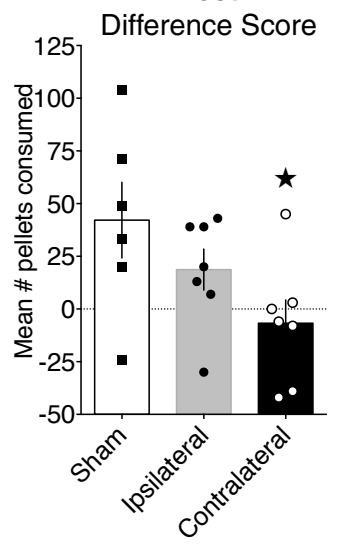

Figure 3. Effect of mPFC-LHA system disconnection on cue-potentiated feeding. $\boldsymbol{A}$, Experimental design. $\boldsymbol{B}$, Representative images of NeuN-stained tissue in lesioned and sham animals. $\boldsymbol{C}$, The extent of mPFC and LHA lesions, shown on modified atlas templates (Swanson, 2004). $\boldsymbol{D}$, Conditioned responses during the first and last training sessions. $\boldsymbol{E}$, Food consumption during cue tests. $\boldsymbol{F}$, Difference in consumption between tests with $C S^{+}$and $C S^{-}$. $D-F$, Data are mean \pm SEM. ${ }^{*} p<0.05$.

0.339). During the cue tests, rats consumed more food during the test with $\mathrm{CS}^{+}$presentations than the test with $\mathrm{CS}^{-}$presentations, indicating cue-potentiated feeding. Figure $3 E$ displays the average pellets consumed during each cue test. A difference score for cue consumption was calculated (the amounts consumed during $\mathrm{CS}^{+}$minus the amounts consumed during $\mathrm{CS}^{-}$tests) and is shown in Figure $3 F$. A one-way ANOVA with orthogonal contrasts revealed that the effect of lesion on test consumption was significant $\left(F_{(2,17)}=3.523, p=0.05\right)$. Contrast testing showed that this measure of potentiation of eating was reduced significantly in the contralateral group compared with the ipsilateral and sham groups $\left(t_{(17)}=2.386, p=0.029\right)$, while this difference in consumption between $\mathrm{CS}^{+}$and $\mathrm{CS}^{-}$tests did not differ significantly between the two control groups $\left(t_{(11)}=1.198, p=\right.$ $0.256)$.
Experiment 4: mapping of the LHA ORX projection to $\mathrm{MPFC}$ In this experiment, retrograde tracing was combined with ORX detection to confirm and better characterize an ascending projection to the mPFC from LHA ORX neurons. The rostrocaudal spread of the FG deposit in the $\mathrm{mPFC}$ of a representative brain is shown in Figure $4 A$. The tracer deposit was primarily contained within the PL and ILA, with minimal spread caudally into the dorsal part of the tenia tecta and indusium griseum. In Figure $4 B$, images of the FG injection site in the mPFC (top left), and examples of retrogradely labeled neurons in the LHA (top middle) are shown. The remaining panels in Figure $4 B$ depict plots of each single- (FG; green) and double-labeled (FG + ORX; magenta) neurons found throughout the LHA. FG-labeled neurons were observed throughout the rostrocaudal extent of the LHA examined (Swanson Atlas Levels 26-32, - 1.78 through $-3.90 \mathrm{~mm}$ 
A

\section{Placement of FG injection in mPFC}

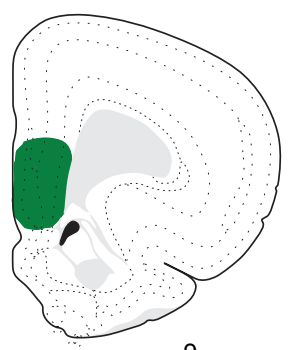

9

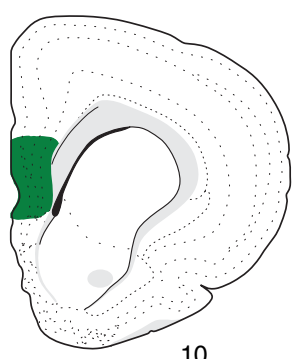

10

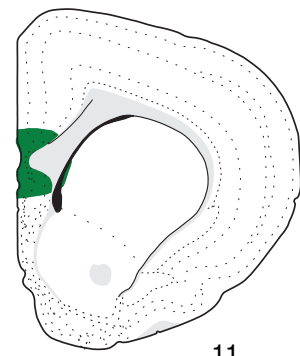

11

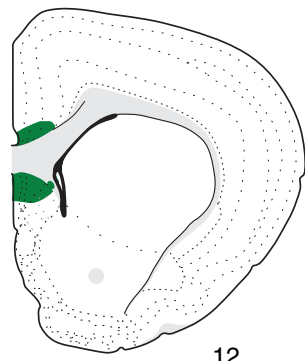

12
B

FG deposit in mPFC
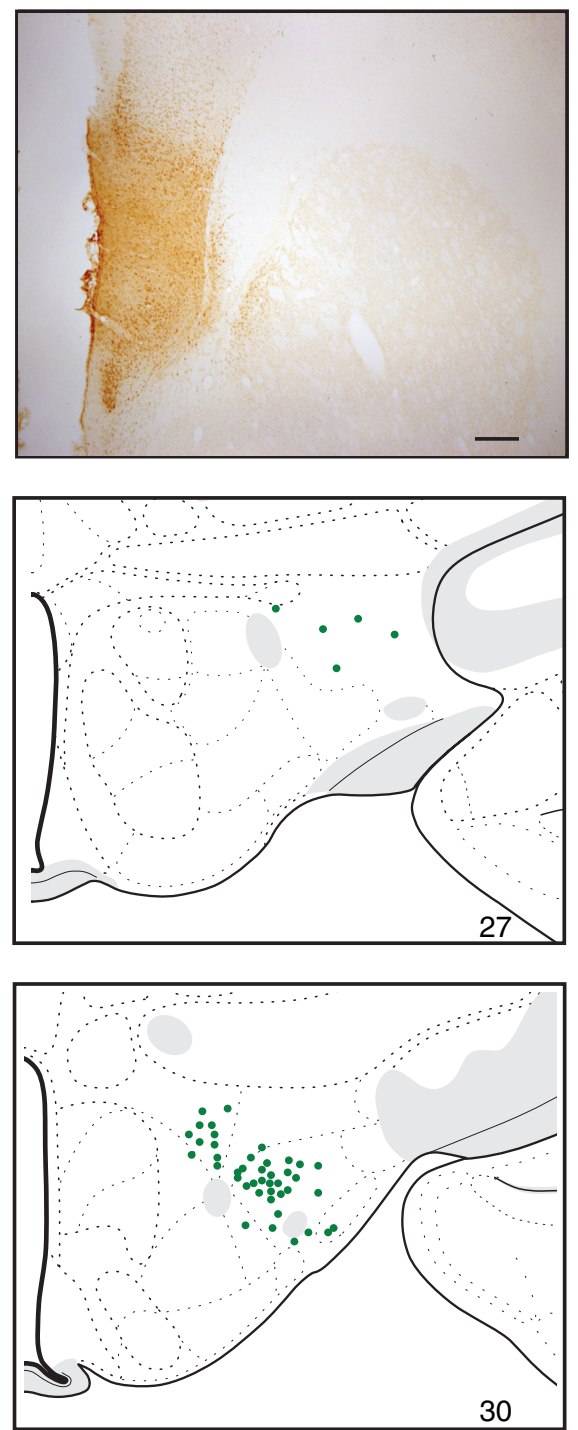

Representative FG and ORX labeling in LHA
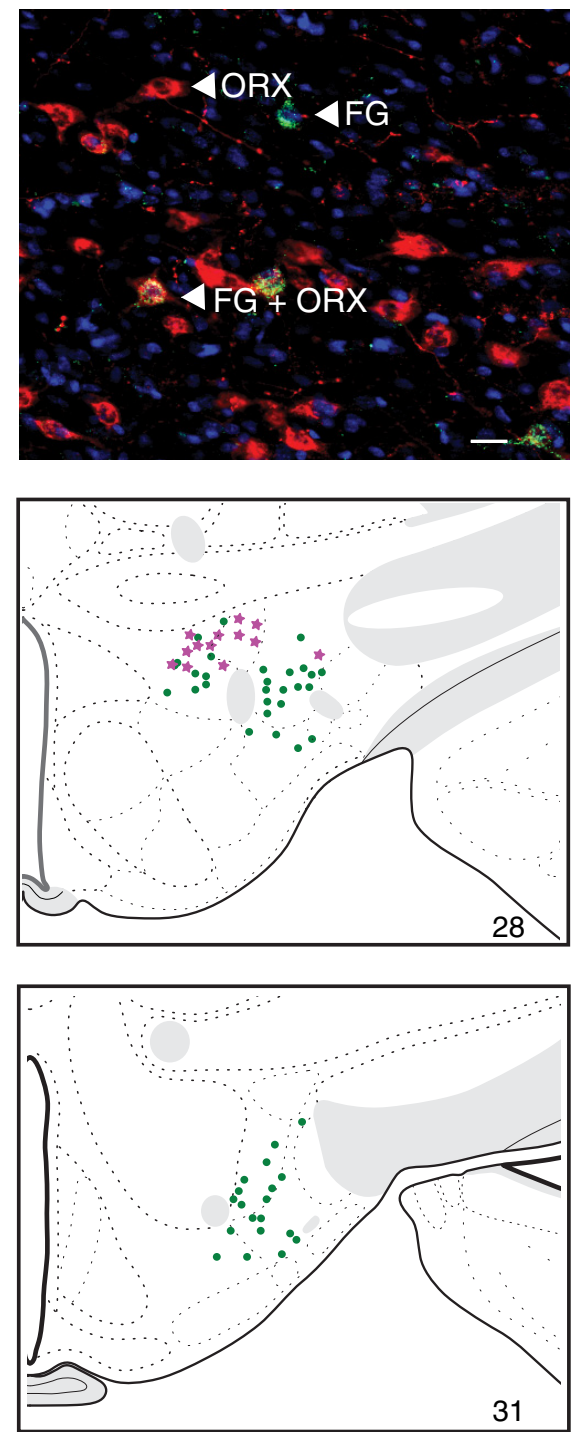

Plots of FG and FG + ORX labeled neurons
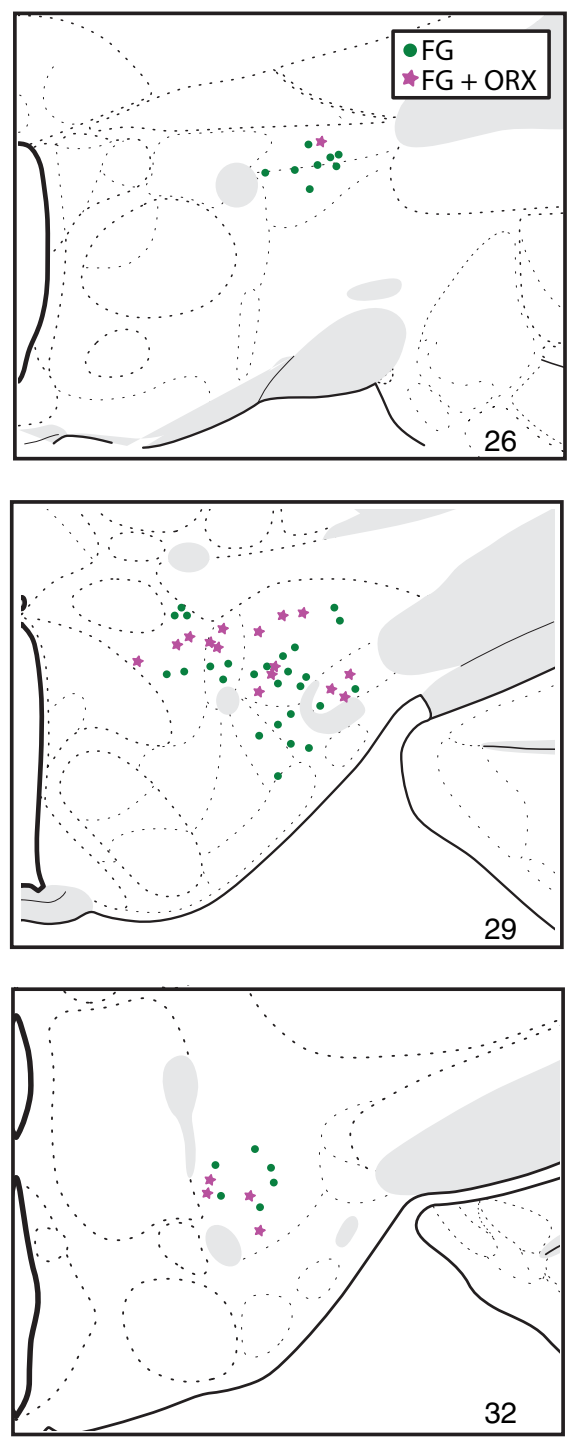

Figure 4. LHA orexinergic projection to mPFC.A, Placement of FG injection in mPFC. B, A representative image of FG depositin mPFC (first panel), scale bar $=100 \mu \mathrm{m}$. A representative image of FG and ORX labeling in LHA (second panel), scale bar $=25 \mu \mathrm{m}$. Plots of FG and FG + ORX labeled neurons, shown on modified atlas templates (Swanson, 2004). Green dots $=$ FG-labeled neurons; Magenta $=$ Double-labeled FG + ORX-positive neurons.

from bregma), but they were densest in the middle levels (28-30, -2.45 through $-3.25 \mathrm{~mm}$ from bregma), an area that corresponds with the densest ORX labeling (Swanson et al., 2005). Double-labeled neurons were also predominantly restricted to levels $28(-2.45 \mathrm{~mm}$ from bregma) and $29(-2.85 \mathrm{~mm}$ from bregma), with fewer in the most rostral and caudal levels (26, $-1.78 \mathrm{~mm}$, and $32,-3.90 \mathrm{~mm}$ from bregma, respectively). The FG + ORX neurons were almost exclusively located dorsal to the 
fornix, with approximate equivalent density across medial and lateral subnuclei. Following quantitative analysis of one representative brain, a total of 166 FGpositive neurons and 501 ORX-positive neurons were observed. Of these neurons, a total of 33 were identified as FG + ORXpositive. Therefore, $\sim 20 \%$ of the LHA neurons identified as the MPFC projecting neurons were ORX neurons, and $\sim 6.5 \%$ of ORX neurons send pathways to the $\mathrm{mPFC}$.

\section{Experiment 5: effect of blocking ORX- $\mathrm{R} 1$ signaling in $\mathrm{MPFC}$ on cue-potentiated feeding}

This experiment examined whether the orexinergic pathway to the $\mathrm{mPFC}$ is causal in cue-potentiated feeding, using intracranial infusions of an ORX 1 receptor antagonist, SB, into mPFC before test. Location of the injector tips in $\mathrm{MPFC}$ are displayed in Figure $5 B$.

\section{Training}

All rats acquired discrimination between cues across training, showing greater foodcup behavior during $\mathrm{CS}^{+}$presentations compared with $\mathrm{CS}^{-}$presentations (Fig. $5 C)$. A group $\times$ training day repeatedmeasures ANOVA showed a main effect of day $\left(F_{(1,12)}=71.792, p<0.001\right)$, but no effect of group $\left(F_{(1,12)}=0.120, p=0.735\right)$ or group $\times$ day interaction $\left(F_{(1,12)}=3.809\right.$, $p=0.075)$.

Test

During the drug-free baseline tests, rats in the SB group consumed fewer pellets. A group $X$ test session repeated-measures ANOVA revealed a main effect of group $\left(F_{(1,12)}=17.336, p=0.001\right)$. However, there was no effect of session $\left(F_{(1,12)}=\right.$ $0.201, p=0.662)$, or group $\times$ session interaction $\left(F_{(1,12)}=0.354, p=0.563\right)$, indicating that while baseline consumption was lower in the SB group, the amount consumed was consistent in both groups across the two baseline tests that preceded $\mathrm{CS}^{+}$and $\mathrm{CS}^{-}$cue tests. During the cue tests, rats that received vehicle consumed more food during the $\mathrm{CS}^{+}$presentations than during $\mathrm{CS}^{-}$presentations, indicating cue-potentiated feeding. Figure $5 D$ displays the average pellets consumed during each cue test. The difference score for cue consumption is shown in Figure 5E. An independent samples $t$ test revealed that this cue-potentiated eating was significantly reduced in rats receiving SB infusion into the $\operatorname{mPFC}\left(t_{(12)}=2.956, p=0.012\right)$.

\section{Discussion}

The current study demonstrated that neuronal ensemble plasticity and ORX-R1 signaling within the $\mathrm{MPFC}$ are necessary in CPF. First, we showed that the $\mathrm{MPFC}$ is activated during the test phase of CPF, and that the neuronal ensemble previously recruited during the acquisition of cue-food learning is required. These results
Behavioral Procedure

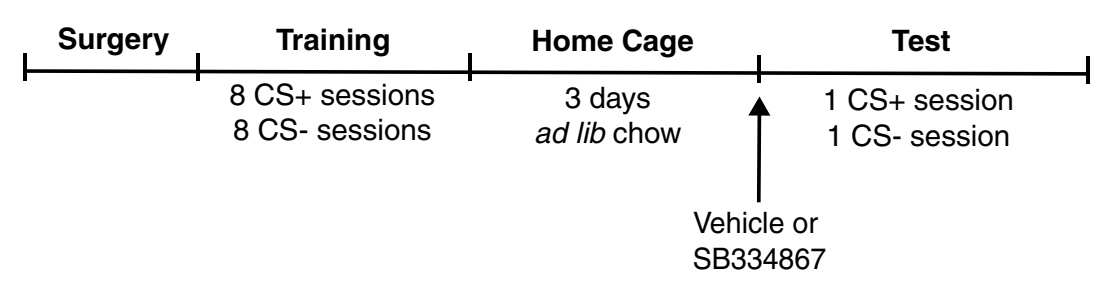

C
Cannula Placements

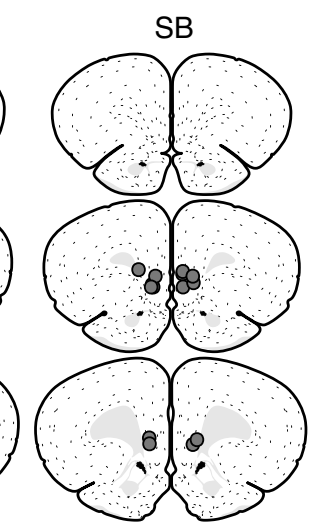

consumption

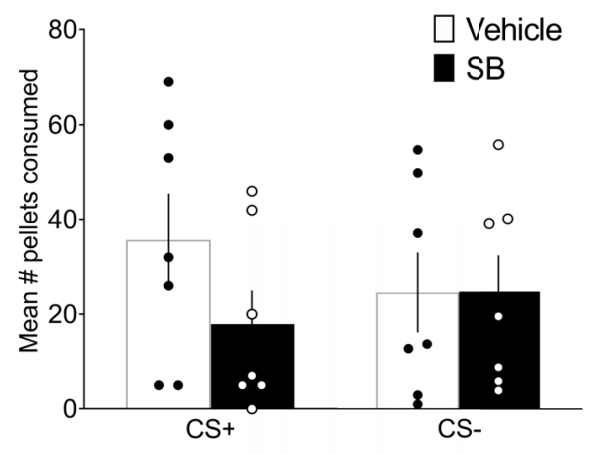

Training Difference Score

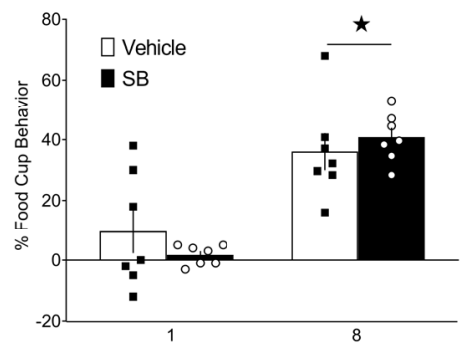

Session
E

Test

Difference Score

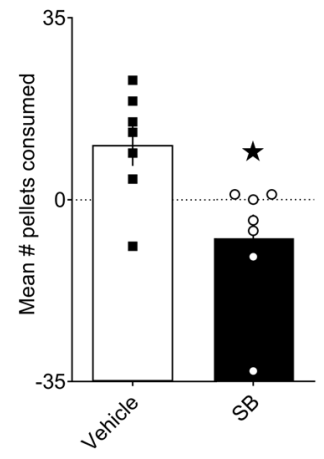

Figure 5. Effect of blocking ORX-R1 signaling in MPFC during cue-potentiated feeding. $\boldsymbol{A}$, Experimental design. $\boldsymbol{B}$, Cannula placements. $\boldsymbol{C}$, Conditioned responses during the first and last training sessions. $\boldsymbol{D}$, Food consumption during cue tests. $\boldsymbol{E}$, Difference in consumption between tests with $C S^{+}$and $C S^{-} . C-E$, Data are mean $\pm S E M .{ }^{*} p<0.05$.

indicate the mPFC is a critical site of plasticity across cue-food learning and cue-induced consumption. Next, we showed that connectivity between the MPFC and LHA is necessary for CPF. Then, we confirmed that LHA ORX neurons project to the $\mathrm{mPFC}$, and ORX-R1 signaling within the $\mathrm{MPFC}$ is needed during the test phase of CPF.

The ability of food cues to control food seeking and consumption is acquired through associative learning, and CPF is based on Pavlovian conditioning. Fundamental knowledge about the plasticity of the neural circuit that governs this process, however, is limited. Earlier work identified core components of the CPF neural circuitry (the BLA, LHA, and mPFC) grounded on neurotoxic lesions and immediate early genes induction patterns (Holland et al., 2001; Petrovich et al., 2002, 2005, 2007). In those studies, lesions occurred before any training, leaving to question which specific circuit and neurotransmitter components are necessary 
during the acquisition of cue-food learning versus cue-induced consumption during CPF test. A later analysis of Fos induction patterns within the forebrain during the acquisition of cue-food learning demonstrated the BLA, LHA, and mPFC are differentially activated across training (Cole et al., 2013, 2015b). The BLA, specifically the anterior basolateral nucleus, was the only forebrain region that showed learning-related Fos induction during the first training session, but at the end of training, when the cue-food association was well established, similar patterns were also evident in the LHA and mPFC. These patterns suggest that the BLA is recruited first and, as the learning progresses, it informs the rest of the circuitry through the BLA-LHA-mPFC connectional network (Reppucci and Petrovich, 2016). Confirming this prediction, more BLA neurons that send direct pathways to the $\mathrm{mPFC}$ were recruited (indicated by Fos induction) when the cue-food association was well learned compared with the control group (Keefer and Petrovich, 2017). Thus, the mPFC is well positioned to access cue-food learning and memory information and impact food motivation accordingly.

Building on this background, the current study demonstrated that $\mathrm{mPFC}$ recruitment is necessary during $\mathrm{CPF}$ tests and is a site of plasticity. First, we showed that Fos induction was higher in mPFC (PL and ILA) during CPF tests, when the food cue drives consumption in the experimental (paired) compared with control (unpaired) conditions. Then, with the Daun02 inactivation method, we targeted the mPFC neurons that were activated during the last cue-food session. Rats that received Daun02 after the last training session were impaired during CPF test compared with the vehicle group, indicating that the same mPFC neuronal ensemble that was activated during cue-food learning needs to be reactivated during cue-driven consumption. These findings are in agreement with prior studies that used the same inactivation method and identified a neuronal ensemble across mPFC that mediates food reward memory and guides food seeking in an operant task (Warren et al., 2016; Whitaker et al., 2017). Thus, the mPFC is necessary to access food cue memory, and we conceptualize its function within a broader role in guiding reward behaviors under cognitive influence, including context-mediated renewal of responding to reward cues (Bossert et al., 2012; for review, see Moorman et al., 2015; Anderson and Petrovich, 2018), and drug self-administration (Rubio et al., 2019).

Next, we showed that mPFC-LHA communications are critical for CPF. We found no impairments in cue-food learning in the mPFC-LHA disconnection group, in agreement with prior work with unilateral LHA manipulations (Petrovich et al., 2002). The disconnection group was impaired during the cue consumption test, potentially because mPFC-LHA communications may be needed to integrate cue memory and feeding motivation (Petrovich, 2018). The mPFC has extensive connections with the LHA, including inputs to the ORX neurons (Sakurai et al., 2005; Yoshida et al., 2006; for a recent review, see Reppucci and Petrovich, 2016) and that pathway may be mediating CPF and was disrupted with the disconnection. As discussed earlier, the LHA is a crucial part of CPF network that is critical during learning and expression of food cue-driven behaviors (Sharpe et al., 2017). Similarly, ORX neurons are recruited during both cue-food learning and cue motivated actions, and blockade of ORX signaling impacts cue-food learning and CPF tests (Harris et al., 2005; Choi et al., 2010; Petrovich et al., 2012; Cole et al., 2015a,b; Keefer et al., 2016). Conversely, disruption of ORX-to-mPFC pathway could have caused CPF impairments with mPFC-LHA disconnection.
The outputs of ORX neurons are exceptionally widespread (de Lecea et al., 1998; Peyron et al., 1998; Sakurai et al., 1998), and specific targets that mediate food motivation in Pavlovian cue driven behaviors have not been delineated. ORX infusions into cortical (orbitofrontal and insular) as well as subcortical (nucleus accumbens and ventral pallidum) areas enhance hedonic reactions to sucrose (Ho and Berridge, 2013; Castro et al., 2016; Castro and Berridge, 2017). Our recent work strongly implicated the mPFC, and ORX signaling there or within its connected areas, based on Fos induction patterns after systemic blockade of ORX-R1 signaling that impaired CPF (Cole et al., 2015a). Anatomical evidence exists that the $\mathrm{MPFC}$ receives direct inputs from LHA, and it contains ORX fibers and receptors (Peyron et al., 1998; Marcus et al., 2001; Hahn and Swanson, 2012, 2015). However, whether ORX neurons send direct inputs to the mPFC had not been confirmed with retrograde tracers. Here, we demonstrated with retrograde labeling that ORX neurons contribute to the LHA-mPFC projections. To test the role of these pathways in $\mathrm{CPF}$, in the last experiment, we infused the ORX-R1 antagonist, SB-334867, into mPFC before tests. These SB infusions abolished cue-driven eating, demonstrating ORX signaling within the $\mathrm{mPFC}$ mediates cue-induced consumption during CPF.

Here, CPF induced Fos within the mPFC, whereas previously systemic ORX-R1 antagonism also induced Fos in mPFC but blocked CPF (Cole et al., 2015a). The most parsimonious explanation would be that Fos expression occurs in different neurons due to ORX signaling and when its receptors are blocked. If ORX activates an inhibitory neuron 1 that synapses on an inhibitory neuron 2, which ends on a pyramidal neuron, that cascade would result in Fos induction in the inhibitory 1 and pyramidal (projecting) neurons. ORX-R1 blockade would cause the opposite: Fos induction in the inhibitory neuron 2 due to a release from inhibition, while the output (pyramidal neuron) would be inhibited. It is also possible that more complex mechanisms mediate ORX action within mPFC: ORX may presynaptically modulate GABA or glutamate release (Scammell and Winrow, 2011; Aracri et al., 2015); while most ORX neurons are glutamatergic, some may release GABA (Bonnavion et al., 2016).

There are four methodological issues that require explanations. First, the mPFC manipulations encompassed PL and ILA and thus could not determine specific contributions of each area. Also, regarding topography, the areas demarcated for Fos counting were slightly different in size and location for Experiments 1 and 2 due to methodological constraints. The template area in Experiment 2 was smaller, and the location was determined by the cannula placement and was centered below the ventral point of the injector tip. Consequently, overall fewer Fos cells were counted in Experiment 2. Second, in Experiment 2, the Fos induction session before Daun02 infusion was a cue-food training session. Another option could have been to induce Fos during the food-cue (tone) alone presentations. However, that would have been an extinction session, and different mPFC neurons have been shown to mediate reward memory versus extinction recall (Warren et al., 2016). Third, the lesions in the disconnection experiment were large and did not selectively target ORX neurons or specific parts of the LHA or mPFC. Another limitation of the lesion disconnection method is that it cannot determine whether the LHA-to-mPFC, mPFC-to-LHA, or both pathways are critical. Fourth, in Experiment 5, the SB dose was high and could have potentially activated ORX-R2 in addition to ORX-R1 (Scammell and Winrow, 2011). Also, there was a difference in consumption during baseline drug-free tests between SB- and vehicle-treated groups. The baseline consumption was lower in the SB group, but 
similar amounts were consumed across the two baseline tests that preceded $\mathrm{CS}^{+}$and $\mathrm{CS}^{-}$cue tests.

In conclusion, our study demonstrated the necessity of mPFC neuronal plasticity and ORX-R1 signaling in cue-induced feeding. These findings are of broad interest, as this form of cognitive motivation to eat is increasingly relevant to overeating in the contemporary world that contains highly palatable foods and their cues (Berthoud, 2012; Petrovich, 2013). Indeed, a metaanalysis confirmed that food cue exposure and associated experience of craving drive eating behavior and weight gain in humans (Boswell and Kober, 2016). Notably, activation of key CPF circuit components in response to food cues in sated humans, the BLA, and its connectivity with the LHA, predicts weight gain over a year (Sun et al., 2015). This work should inspire future studies determining network and molecular mediators underlying individual differences in vulnerability to compulsive food seeking and overeating, ultimately leading to new treatments and prevention of excessive food drive.

\section{References}

Anderson LC, Petrovich GD (2018) Ventromedial prefrontal cortex mediates sex differences in persistent cognitive drive for food. Sci Rep 8:2230.

Aracri P, Banfi D, Pasini ME, Amadeo A, Becchetti A (2015) Hypocretin (Orexin) regulates glutamate input to fast-spiking interneurons in layer $\mathrm{V}$ of the Fr2 region of the murine prefrontal cortex. Cereb Cortex 25:13301347.

Balleine B, O'Doherty JP (2010) Human and rodent homologies in action control: corticostriatal determinants of goal directed and habitual action. Neuropsychopharmacology 35:48-69.

Berthoud HR (2012) The neurobiology of food intake in an obesogenic environment. Proc Nutr Soc 71:478-487.

Birch LL, McPhee L, Sullivan S, Johnson S (1989) Conditioned meal initiation in young children. Appetite 13:105-113.

Bonnavion P, Mickelsen LE, Fujita A, de Lecea L, Jackson AC (2016) Hubs and spokes of the lateral hypothalamus: cell types, circuits and behaviour. J Physiol 594 22:6443-6462.

Borgland SL, Taha SA, Sarti F, Fields HL, Bonci A (2006) Orexin A in the VTA is critical for the induction of synaptic plasticity and behavioral sensitization to cocaine. Neuron 49:589-601.

Bossert JM, Stern AL, Theberge FR, Marchant NJ, Wang HL, Morales M, Shaham Y (2012) Role of projections from ventral medial prefrontal cortex to nucleus accumbens shell in context-induced reinstatement of heroin seeking. J Neurosci 32:4982-4991.

Boswell RG, Kober H (2016) Food cue reactivity and craving predict eating and weight gain: a meta-analytic review. Obes Rev 17:159-177.

Castro DC, Berridge KC (2017) Opioid and orexin hedonic hotspots in rat orbitofrontal cortex and insula. Proc Natl Acad Sci U S A 114:E9125E9134.

Castro DC, Terry RA, Berridge KC (2016) Orexin in rostral hotspot of nucleus accumbens enhances sucrose 'liking' and intake but scopolamine in caudal shell shifts 'liking' toward 'disgust' and 'fear.' Neuropharmacology 41:2101-2111.

Choi DL, Davis JF, Fitzgerald ME, Benoit SC (2010) The role of orexin-A in food motivation, reward-based feeding behavior and food-induced neuronal activation in rats. Neuroscience 167:11-20.

Cole S, Powell DJ, Petrovich GD (2013) Differential recruitment of distinct amygdalar nuclei across appetitive associative learning. Learn Mem 20: 295-299.

Cole S, Mayer HS, Petrovich GD (2015a) Orexin/hypocretin-1 receptor antagonism selectively reduces cue-induced feeding in sated rats and recruits medial prefrontal cortex and thalamus. Sci Rep 5:16143.

Cole S, Hobin MP, Petrovich GD (2015b) Appetitive associative learning recruits a distinct network with cortical, striatal, and hypothalamic regions. Neuroscience 286:187-202.

Cornell CE, Rodin J, Weingarten H (1989) Stimulus-induced eating when satiated. Physiol Behav 45:695-704.

Cruz FC, Koya E, Guez-Barber DH, Bossert JM, Lupica CR, Shaham Y, Hope BT (2013) New technologies for examining the role of neuronal ensembles in drug addiction and fear. Nat Rev Neurosci 14:743-754.

de Lecea L, Kilduff TS, Peyron C, Gao X, Foye PE, Danielson PE, Fukuhara C,
Battenberg EL, Gautvik VT, Bartlett FS 2nd, Frankel WN, van den Pol AN, Bloom FE, Gautvik KM, Sutcliffe JG (1998) The hypocretins: hypothalamus-specific peptides with neuroexcitatory activity. Proc Natl Acad Sci U S A 95:322-327.

Fanous S, Goldart EM, Theberge FR, Bossert JM, Shaham Y, Hope BT (2012) Role of orbitofrontal cortex neuronal ensembles in the expression of incubation of heroin craving. J Neurosci 32:11600-11609.

Funk D, Coen K, Tamadon S, Hope BT, Shaham Y, Lê AD (2016) Role of central amygdala neuronal ensembles in incubation of nicotine craving. J Neurosci 36:8612-8623.

Hahn JD, Swanson LW (2012) Connections of the lateral hypothalamic area juxtadorsomedial region in the male rat. J Comp Neurol 520:1831-1890.

Hahn JD, Swanson LW (2015) Connections of the juxtaventromedial region of the lateral hypothalamic area in the male rat. Front Syst Neurosci 9:66.

Harris GC, Wimmer M, Aston-Jones G (2005) A role for lateral hypothalamic orexin neurons in reward seeking. Nature 437:556-559.

Ho CY, Berridge KC (2013) An orexin hotspot in ventral pallidum amplifies hedonic 'liking' for sweetness. Neuropsychopharmacology 38:1655-1664.

Holland PC, Hatfield T, Gallagher M (2001) Rats with basolateral amygdala lesions show normal increases in conditioned stimulus processing but reduced conditioned potentiation of eating. Behav Neurosci 115:945-950.

James MH, Charnley JL, Levi EM, Jones E, Yeoh JW, Smith DW, Dayas CV (2011) Orexin-1 receptor signalling within the ventral tegmental area, but not the paraventricular thalamus, is critical to regulating cue-induced reinstatement of cocaine-seeking. Int J Neuropsychopharmacol 14:684-690.

Keefer SE, Petrovich GD (2017) Distinct recruitment of basolateral amygdala-medial prefrontal cortex pathways across Pavlovian appetitive conditioning. Neurobiol Learn Mem 141:27-32.

Keefer SE, Cole S, Petrovich GD (2016) Orexin/hypocretin receptor 1 signaling mediates Pavlovian cue-food conditioning and extinction. Physiol Behav 162:27-36.

Koya E, Margetts-Smith G, Hope BT (2016) Daun02 inactivation of behaviorally activated Fos-expressing neuronal ensembles. Curr Protoc Neurosci 76:8.36.1-38.36.17.

Land BB, Narayanan NS, Liu RJ, Gianessi CA, Brayton CE, Grimaldi DM, Sarhan M, Guarnieri DJ, Deisseroth K, Aghajanian GK, DiLeone RJ (2014) Medial prefrontal D1 dopamine neurons control food intake. Nat Neurosci 17:248-253.

Mahler SV, Moorman DE, Smith RJ, James MH, Aston-Jones G (2014) Motivational activation: a unifying hypothesis of orexin/hypocretin function. Nat Neurosci 17:1298-1303.

Marcus JN, Aschkenasi CJ, Lee CE, Chemelli RM, Saper CB, Yanagisawa M, Elmquist JK (2001) Differential expression of orexin receptors 1 and 2 in the rat brain. J Comp Neurol 435:6-25.

Mena JD, Sadeghian K, Baldo BA (2011) Induction of hyperphagia and carbohydrate intake by $\mu$-opioid receptor stimulation in circumscribed region of frontal cortex. J Neurosci 31:3249-3260.

Mena JD, Selleck RA, Baldo BA (2013) Mu-opioid stimulation in rat prefrontal cortex engages hypothalamic orexin/hypocretin-containing neurons, and reveals dissociable roles of nucleus accumbens and hypothalamus in cortically driven feeding. J Neurosci 33:18540-18552.

Moorman DE, James MH, McGlinchey EM, Aston-Jones G (2015) Differential roles of medial prefrontal subregions in the regulation of drug seeking. Brain Res 1628:130-146.

O'Doherty JP (2011) Contributions of the ventromedial prefrontal cortex to goal-directed action selection. Ann N Y Acad Sci 1239:118-129.

Petrovich GD (2013) Forebrain networks and the control of feeding by environmental learned cues. Physiol Behav 121:10-18.

Petrovich GD (2018) Lateral hypothalamus as a motivation-cognition interface in the control of feeding behavior. Front Syst Neurosci 12:14.

Petrovich GD, Setlow B, Holland PC, Gallagher M (2002) Amygdalohypothalamic circuit allows learned cues to override satiety and promote eating. J Neurosci 22:8748-8753.

Petrovich GD, Holland PC, Gallagher M (2005) Amygdalar and prefrontal pathways to the lateral hypothalamus are activated by a learned cue that stimulates eating. J Neurosci 25:8295-8302.

Petrovich GD, Ross CA, Holland PC, Gallagher M (2007) Medial prefrontal cortex is necessary for an appetitive contextual conditioned stimulus to promote eating in sated rats. J Neurosci 27:6436-6441.

Petrovich GD, Hobin MP, Reppucci CJ (2012) Selective Fos induction in hypothalamic orexin/hypocretin, but not melanin-concentrating hor- 
mone neurons, by a learned food-cue that stimulates feeding in sated rats. Neuroscience 224:70-80.

Peyron C, Tighe DK, van den Pol AN, de Lecea L, Heller HC, Sutcliffe JG, Kilduff TS (1998) Neurons containing hypocretin (orexin) project to multiple neuronal systems. J Neurosci 18:9996-10015.

Reppucci CJ, Petrovich GD (2016) Organization of connections between the amygdala, medial prefrontal cortex, and lateral hypothalamus: a single and double retrograde tracing study in rats. Brain Struct Funct 221: 2937-2962.

Rubio FJ, Quintana-Feliciano R, Warren BL, Li X, Witonsky KF, Valle FS, Selvam PV, Caprioli D, Venniro M, Bossert JM, Shaham Y, Hope BT (2019) Prelimbic cortex is a common brain area activated during cueinduced reinstatement of cocaine and heroin seeking in a polydrug selfadministration rat model. Eur J Neurosci 49:165-178.

Sakurai T (2014) The role of orexin in motivated behaviours. Nat Rev Neurosci 15:719-731.

Sakurai T, Amemiya A, Ishii M, Matsuzaki I, Chemelli RM, Tanaka H, Williams SC, Richardson JA, Kozlowski GP, Wilson S, Arch JR, Buckingham RE, Haynes AC, Carr SA, Annan RS, McNulty DE, Liu WS, Terrett JA, Elshourbagy NA, Bergsma DJ, et al. (1998) Orexins and orexin receptors: a family of hypothalamic neuropeptides and G protein-coupled receptors that regulate feeding behavior. Cell 92:573-585.

Sakurai T, Nagata R, Yamanaka A, Kawamura H, Tsujino N, Muraki Y, Kageyama H, Kunita S, Takahashi S, Goto K, Koyama Y, Shioda S, Yanagisawa M (2005) Input of orexin/hypocretin neurons revealed by a genetically encoded tracer in mice. Neuron 46:297-308.

Scammell TE, Winrow CJ (2011) Orexin receptors: pharmacology and therapeutic opportunities. Annu Rev Pharmacol Toxicol 51:243-266.

Sharpe MJ, Marchant NJ, Whitaker LR, Richie CT, Zhang YJ, Campbell EJ, Koivula PP, Necarsulmer JC, Mejias-Aponte C, Morales M, Pickel J, Smith JC, Niv Y, Shaham Y, Harvey BK, Schoenbaum G (2017) Lateral hypothalamic GABAergic neurons encode reward predictions that are relayed to the ventral tegmental area to regulate learning. Curr Biol 27:2089-2100.e5.

Smith RJ, Laiks LS (2018) Behavioral and neural mechanisms underlying habitual and compulsive drug seeking. Prog Neuropsychopharmacol Biol Psychiatry 87:11-21.

Sun X, Kroemer NB, Veldhuizen MG, Babbs AE, de Araujo IE, Gitelman DR, Sherwin RS, Sinha R, Small DM (2015) Basolateral amygdala response to food cues in the absence of hunger is associated with weight gain susceptibility. J Neurosci 35:7964-7976.

Swanson LW (2004) Brain maps: structure of the rat brain. In: A laboratory guide with printed and electronic templates for data, models and schematics, Ed 3 (revised with CD-ROM). Amsterdam: Elsevier.

Swanson LW, Sanchez-Watts G, Watts AG (2005) Comparison of melaninconcentrating hormone and hypocretin/orexin mRNA expression patterns in a new parceling scheme of the lateral hypothalamic zone. Neurosci Lett 387:80-84.

Warren BL, Mendoza MP, Cruz FC, Leao RM, Caprioli D, Rubio FJ, Whitaker LR, McPherson KB, Bossert JM, Shaham Y, Hope BT (2016) Distinct Fos-expressing neuronal ensembles in the ventromedial prefrontal cortex mediate food reward and extinction memories. J Neurosci 36:6691-6703.

Weingarten HP (1983) Conditioned cues elicit feeding in sated rats: a role for learning in meal initiation. Science 220:431-433.

Whitaker LR, Warren BL, Venniro M, Harte TC, McPherson KB, Beidel J, Bossert JM, Shaham Y, Bonci A, Hope BT (2017) Bidirectional modulation of intrinsic excitability in rat prelimbic cortex neuronal ensembles and non-ensembles after operant learning. J Neurosci 37:8845-8856.

Yoshida K, McCormack S, España RA, Crocker A, Scammell TE (2006) Afferents to the orexin neurons of the rat brain. J Comp Neurol 494:845861. 